\title{
Rainbow Gravity: Big Bounce in Bianchi Type I Universe
}

\author{
Wesley Wong ${ }^{1, *}$, Chee Leong Ching ${ }^{1, * *}$ and Wei Khim $\mathrm{Ng}^{1, * * *}$ \\ ${ }^{1}$ Physics Department, National University of Singapore, 2 Science Drive 3, Singapore 117551
}

\begin{abstract}
We will examine the Bianchi Type I universe under the Rainbow Gravity formalism and calculate various quantities like the dynamical equation for the energy density and the negative energy density. Finally, we apply the analysis to a specific Rainbow Gravity model.
\end{abstract}

\section{Introduction}

Rainbow Gravity is a phenomenological theory of quantum gravity. It was proposed by Maguejio and Smolin [1] to solve some puzzles in the cosmological observations like the threshold anomalies in the ultra high energy cosmic rays and $\mathrm{TeV}$ photons. Further studies have shown that certain Rainbow Gravity models predict a Big Bounce which give plausible resolution to the problem of Big Bang singularity [2-4].

Recent high-precision cosmological observations indicate anisotropies in the Cosmic Microwave Background (CMB) [5]. This suggests that the early universe might be anisotropic. The simplest model to desribe this is the Bianchi Type I model.

In this paper, we will probe the early Bianchi Type I universe under the effect of Rainbow Gravity model. In the next section, we will give a brief overview of the Rainbow Gravity model and the Bianchi Type I model. In section 3, we explore the modification of the Bianchi Type I model under the Rainbow Gravity formalism and obtain the general solution of the directional Hubble parameters. In section 4, we examine the Big Bounce solution and probe a specific Rainbow Gravity model [2,3]. Finally, we end with a conclusion and the possible future works.

\section{Background}

\subsection{Rainbow Gravity (RG)}

Under the RG model, the background spacetime observed by the probe particle depends on it's energy. This leads to a modification to the energy dispersion relation given by

$$
E^{2} f^{2}\left(E / E_{p}\right)-p^{2} g^{2}\left(E / E_{p}\right)=m_{0}^{2}
$$

where $E, p$ and $m_{0}$ are the energy, momentum and rest mass of the probe particle, respectively. Here $f^{2}\left(E / E_{p}\right)$ and $g^{2}\left(E / E_{p}\right)$ are some rainbow functions that depends on the energy $E$.

\footnotetext{
*e-mail: a0126599@u.nus.edu

**e-mail: phyccl@nus.edu.sg

***e-mail: phynwk@nus.edu.sg
} 
We have expressed the energy by a factor of the Planck's energy $E_{p}$ to render the rainbow functions dimensionless.

The Einstein's field equations are modified to be

$$
G_{\mu \nu}(E)=8 \pi G(E) T_{\mu \nu}(E)+g_{\mu \nu} \Lambda(E)
$$

where $G(E)$ and $\Lambda(E)$ are the modified Newtonian gravitational constant and cosmological constant, respectively. They can be expressed as $\lambda(E)=h_{1}^{2}(E) \Lambda_{0}$ and $G(E)=h_{2}^{2}(E) G_{0}$, where $G_{0}$ and $\Lambda_{0}$ are the conventional Newtonian gravitational and cosmological constants, respectively. Again, $h_{1}^{2}(E)$ and $h_{2}^{2}(E)$ are some rainbow functions. In the low energy limit $(E \ll 1)$, the rainbow functions $f^{2}, g^{2}, h_{1}^{2}$ and $h_{2}^{2}$ become unity.

\subsection{Bianchi Type I (BI) universe}

The BI model describes a spatially flat homogeneous and anisotropic universe. The line element is given by $d s^{2}=-d t^{2}+a_{1}^{2}(t) d x_{1}^{2}+a_{2}^{2}(t) d x_{2}^{2}+a_{3}^{2}(t) d x_{3}^{2}$ where $a_{i}$ 's are the time dependent scale factors. The standard BI Friedmann-Robertson-Walker (FRW) equations are given by

$$
\begin{aligned}
H_{1} H_{2}+H_{2} H_{3}+H_{3} H_{1} & =8 \pi G_{0} \rho+\Lambda_{0}, \\
\dot{H}_{i}+\dot{H}_{j}+H_{i}^{2}+H_{j}^{2}+H_{i} H_{j} & =-8 \pi G_{0} P+\Lambda_{0} .
\end{aligned}
$$

Here $i, j=1,2,3$ and $i \neq j . \rho$ and $P$ are the energy density and the pressure of perfect fluids, respectively. We have defined the directional Hubble parameters as $H_{i}=\dot{a}_{i} / a_{i}$.

\section{Modified Bianchi Type I universe under the Rainbow Gravity formalism}

The line element for a spatially flat homogeneous and anisotropic universe under the RG formalism is given by

$$
d s^{2}=-\frac{1}{f^{2}(E)} d t^{2}+\sum_{i=1,2,3} \frac{a_{i}^{2}(t)}{g^{2}(E)} d x_{i}^{2}
$$

We define $\mathscr{F}=\dot{f} / f$ and $a_{i, e}=a_{i} / g$. The Hubble parameter can be rewritten as $H_{i, e}=\dot{a}_{i, e} / a_{i, e}$. The Rainbow BI FRW equations become

$$
\begin{aligned}
H_{1, e} H_{2, e}+H_{2, e} H_{3, e}+H_{3, e} H_{1, e} & =\frac{8 \pi h_{2}^{2}}{f^{2}} G_{0} \rho+\frac{h_{1}^{2}}{f^{2}} \Lambda_{0}, \\
\dot{H}_{i, e}+\dot{H}_{j, e}+H_{i, e}^{2}+H_{j, e}^{2}+\mathscr{F}\left(H_{i, e}+H_{j, e}\right) & =-\frac{8 \pi h_{2}^{2}}{f^{2}} G_{0} P+\frac{h_{1}^{2}}{f^{2}} \Lambda_{0},
\end{aligned}
$$

where $i, j=1,2,3$ and $i \neq j$.

The general solution of the directional Hubble parameter $H_{i, e}$ is given by

$$
H_{i, e}(t)=\frac{1}{\mu(t)}\left\{K_{i}+\int \mu(t)\left[\frac{4 \pi h_{2}^{2} G_{0}(\rho-P)}{f^{2}}+\frac{h_{1}^{2} \Lambda_{0}}{f^{2}}\right] d t\right\}
$$

where $\mu=\exp \left[\int_{t_{0}}^{t}\left(3 H_{e}\left(t^{\prime}\right)+\mathscr{F}\left(t^{\prime}\right)\right) d t^{\prime}\right]$. Here $K_{i}$ 's are the integration constants. We can see that the $K_{i}$ 's are the origin of the anisotropy. They are constraint by $K_{1}+K_{2}+K_{3}=0$. Note that $H_{e}=\left(H_{1, e}+H_{2,3}+H_{3, e}\right) / 3$. 
The Bianchi Identity gives a modified local energy conservation as follows

$$
\dot{\rho}+\sum_{i=1,2,3} H_{i, e}(\rho+P)=-\frac{\dot{h}_{1} h_{1} \Lambda_{0}}{8 \pi h_{2}^{2} G_{0}}-\frac{2 \dot{h}_{2} h_{2}}{h_{2}^{2}} \rho .
$$

Note that certain cosmological models has the relation $-\frac{\dot{h}_{1} h_{1} \Lambda_{0}}{8 \pi h_{2}^{2} G_{0}}-\frac{2 \dot{h}_{2} h_{2}}{h_{2}^{2}} \rho=0$. These models satisfy both the Bianchi Identity and the energy conservation.

By substituting the general solution (8) into the Shear Scalar $\sigma^{2}=\frac{3}{2} \sum_{i=1,2,3}\left(H_{i, e}^{2}-H_{e}^{2}\right)$, we obtain the dynamical equation for $\rho$. It is given by

$$
\begin{aligned}
& \frac{1}{24 \pi G_{0}(1+\omega)^{2}}\left[1+\rho_{\Lambda} \frac{\partial_{\rho}\left(h_{1}^{2}\right)}{h_{1}^{2}}+\frac{\partial\left(h_{2}^{2}\right)}{h_{2}^{2}} \rho\right]^{2}\left(\frac{\dot{\rho}}{\rho}\right)^{2}= \\
& \frac{h_{2}^{2} \rho+h_{1}^{2} \rho_{\Lambda}}{\mathscr{F}^{2}}+K_{0}^{2}\left(\frac{f_{0}^{2}}{f^{2}}\right)\left[\left(\frac{\rho}{\rho_{0}}\right)\left(\frac{h_{2}^{2}}{h_{2,0}^{2}}\right) \exp \left(\rho_{\Lambda} \int \frac{\partial_{\rho}\left(h_{1}^{2}\right)}{h_{2}^{2} \rho} d \rho\right)\right]^{2 /(1+\omega)},
\end{aligned}
$$

where the vacuum energy is $\rho_{\Lambda}=\left(\Lambda_{0}\right) /\left(8 \pi G_{0}\right)$ and $K_{0}=-\left(K_{1} K_{2}+K_{2} K_{3}+K_{3} K_{1}\right) /\left(8 \pi G_{0}\right)$. The subscripts " 0 " in $h_{i}$ 's, $f$ 's and $\rho$ 's are at time $t=t_{0}$.

\section{Big Bounce Solution}

We assume there exist a bounded maximum $\rho_{\max }$ (at the Big Bounce) and let $\rho_{0}=\rho_{\max }$. That is the universe contracts until it reaches the threshold $\rho_{\max }$ and bounce back (the collapse to the singularity is avoided). The bounded maximum $\rho_{\max }$ exist at $\dot{\rho}=0$. At $t=t_{0}$, Eq. (10) becomes

$$
\frac{h_{2,0}^{2} \rho_{0}+h_{1,0}^{2} \rho_{\Lambda}}{f_{0}^{2}}+K_{0}^{2}=0 .
$$

The negative vacuum energy density $\rho_{\Lambda}$ is then

$$
\rho_{\Lambda}=-\left(\frac{h_{2,0}^{2}}{h_{1,0}^{2}} \rho_{0}+\frac{f_{0}^{2}}{h_{1,0}^{2}} K_{0}^{2}\right) .
$$

Now, we consider a specific RG model [2, 3] given by

$$
f^{2}=\frac{1}{1-\lambda \rho} \quad, \quad h_{1}^{2}=1+\lambda \rho \quad, \quad h_{2}^{2}=1-\lambda \rho,
$$

where $\lambda$ is some parameter. The negative vacuum energy density and the integration constant are given by

$$
\rho_{\Lambda}=-\frac{1-\lambda}{\lambda} \quad \text { and } \quad K_{0}^{2}=\frac{(1-\lambda)^{2}}{\lambda}
$$

respectively.

The following shows the plot of $\rho$ against time where we let $0<\lambda<1$ and $\omega=1 / 3$ for radiation dominated universe. It shows that small $\lambda$ (higher $\left|\rho_{\Lambda}\right|$ and $K_{0}^{2}$ ) will cause $\rho$ to decay faster (faster volume expansion). 


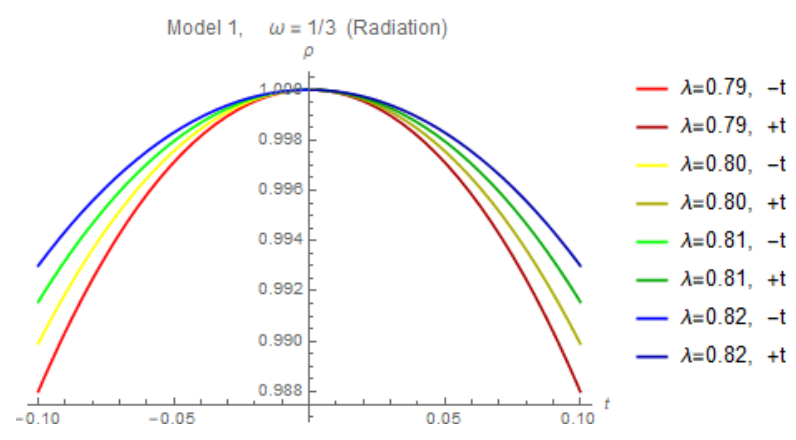

Figure 1: Plot of $\rho$ against time.

Next we plot $\dot{\rho}$ against $\rho$ with the same parameters as in the previous plot.

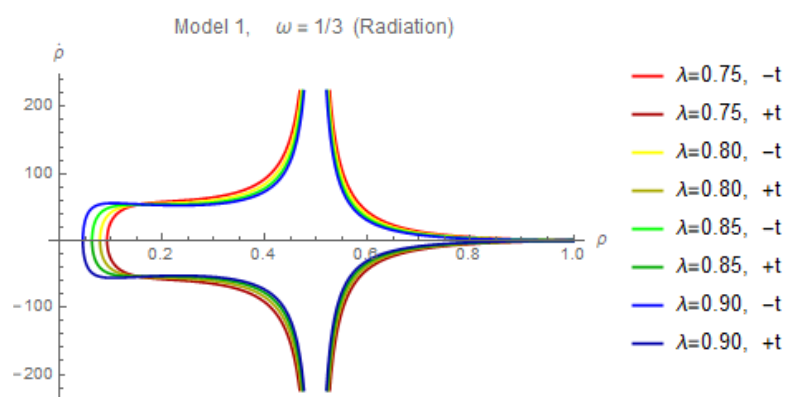

Figure 2: Plot of $\dot{\rho}$ against $\rho$.

Here $\dot{\rho}$ diverges at $\rho=0.5$. This is due to the modified energy conservation. It shows that right after the Big Bounce, the radiation particles will observe a "pseudo-contracting" universe. It could be explained by the plausible scenario that the universe's expansion rate at the beginning is less than the speed of light.

\section{Conclusion}

We have examined the BI universe under the RG formalism and obtained the modified FRW equations. We also derived the dynamical equation for the energy density and the negative energy density needed to satisfy the Big Bounce. Finally, for a specific Rainbow model, we have shown that the large anisotropy cause the energy density to decay faster and there exist a "pseudo-contracting" universe after the Big Bounce. For future work, we can compare other Rainbow Gravity models and attempt to fit them with current observations.

\section{References}

[1] J. Magueijo and L. Smolin, Class. Quant. Grav., 21, 1725 (2004)

[2] Y. Ling, JCAP, 0708, 017 (2007)

[3] Y. Ling and Q. Wu, Phys. Lett. B, 687, 103 (2010)

[4] A. Awad, A. F. Ali and B. Majumder, JCAP, 1310, 052 (2013)

[5] E. Russell, C. B. Kilinc and O. K. Pashaev, Mon. Not. Roy. Astron. Soc., 442, no. 3, 2331 (2014) 